\title{
Editorial: The Fungal Cell Wall
}

\author{
Fausto Almeida ${ }^{1 *}$, Joshua D. Nosanchuk ${ }^{2,3 *}$ and Gustavo Alexis Niño-Vega ${ }^{4 *}$ \\ 1 Department of Biochemistry and Immunology, Ribeirão Preto Medical School, University of São Paulo, São Paulo, Brazil, \\ ${ }^{2}$ Division of Infectious Diseases, Department of Medicine, Albert Einstein College of Medicine, New York, NY, United States, \\ ${ }^{3}$ Department of Microbiology, Albert Einstein College of Medicine, New York, NY, United States, ${ }^{4}$ Division de Ciencias \\ Naturales y Exactas, Departamento de Biologia, Universidad de Guanajuato, Guanajuato, Mexico
}

Keywords: cell wall, fungi, pathogenic fungi, pathogenic fungal disease, non-pathogenic fungi, fungal structure, fungal metabolism

\section{Editorial on the Research Topic}

\section{The Fungal Cell Wall}

A robust understanding of the complexity and functionality of the fungal cell wall is crucial to the development of new therapeutic and prophylactic strategies. The cell wall plays several key functions in fungal pathobiology as diverse factors, such as cell shape, encapsulation, and rigidity, influence events during interaction with the host (Gow et al., 2017). These interactions may be proinflammatory or may subvert host responses. The fungal cell wall has a flexible structure that is highly complex and intricately organized of $\alpha$ - and $\beta$ - linked glucans, chitin, glycoproteins, and pigments (Gow et al., 2017).

The researchers who contributed to this Research Topic presented 13 themed articles that Gustavo Henrique Goldman University of São Paulo, Brazil

Reviewed by: Carol Munro, University of Aberdeen,

United Kingdom Iran Malavazi,

Federal University of São Carlos, Brazil

${ }^{*}$ Correspondence:

Fausto Almeida

fbralmeida@usp.br

Joshua D. Nosanchuk

josh.nosanchuk@einsteinmed.org

Gustavo Alexis Niño-Vega

gustavo.nino@ugto.mx

Specialty section

This article was submitted to

Fungi and Their Interactions,

a section of the journal

Frontiers in Microbiology

Received: 18 May 2020

Accepted: 26 June 2020

Published: 09 September 2020

Citation:

Almeida F, Nosanchuk JD and Niño-Vega GA (2020) Editorial: The

Fungal Cell Wall.

Front. Microbiol. 11:1682.

doi: 10.3389/fmicb.2020.01682 highlighted the latest advances in our understanding of the biological importance of the fungal cell wall. For example, Garcia-Rubio et al. summarized recent findings on the characteristics and influence of cell wall components on fungi-host interaction with a specific focus on three fungal species, Aspergillus fumigatus, Candida albicans, and Cryptococcus neoformans. Lima et al. discussed the wealth of information available regarding antifungal therapy and the development of antimicrobial resistance subsequent to cell wall modifications by several important human pathogenic fungi. Patel and Free addressed the genetics and biochemistry that lead to the formation of the complex Neurospora crassa cell wall, comparing this species' cell wall with that of other fungal species.

Two reports focused on the dermatophyte Trichophyton rubrum. Martins et al. evaluated RNAseq results under stress conditions, using undecanoic acid and acriflavine as well as the influence of the carbon source, on the modulation of genes regulating T. rubrum cell wall metabolism. These investigators described that keratin mimics the host environment and undecanoic acid and acriflavine present non-specific antifungal activity against T. rubrum. Thus, the authors identified genes putatively encoding T. rubrum virulence factors. Celestrino et al. verified that Tolllike receptor 2 (TLR2) is required for efficient phagocytosis of T. rubrum conidia by adherent monocytes, and the absence of TLR2 signaling in human monocytes impairs the expected inflammatory responses.

In the work by Sun et al., we learnt that the farnesyltranferase $\beta$ subunit Ram 1 regulates pathogenicity, mating, and cell wall integrity, and it also plays an important role as a virulence factor in the sugarcane smut fungus Sporisorium scitamineum. Miyazawa et al. described that both $\alpha$-1,3-glucan and galactosaminogalactan are adhesive molecules and these glucans contribute to aggregation on the hyphal surface of Aspergillus oryzae. In P. brasiliensis, Souza et al. verified that cell wall $\alpha$-glucan induced differentiation of dendritic cells, which could contribute to pathogen persistence since this process potentially affects Th1 polarization. de Oliveira et al. demonstrated that thioredoxin reductase 1 is a highly immunogenic surface antigen in the cell walls of 
Candida albicans, Paracoccidioides spp., and Cryptococcus neoformans, and that the enzyme has conserved epitopes in fungi, but there are no homologs in humans. In Candida parapsilosis, Oh et al. verified the nature of the agglutinin-like sequence gene family, which encodes cell-surface glycoproteins involved in the adhesion of fungal cells to host and abiotic surfaces. The authors also demonstrated allelic variability and expression patterns. The Zheng et al. verified that the deletion of a gene responsible for coding a calcineurin homolog from Talaromyces marneffe $i$ affects germination, cell wall integrity, morphogenesis, and resistance to external stresses. Orner et al. demonstrated that the cellwall-associated antiphagocytic protein 1 and laccase enzymes (named Lac1 and Lac2) play important roles in increasing resistance to amphotericin $\mathrm{B}$ and host-mediated killing during infection as well as enhancing the subsequent accumulation of old C. neoformans cells (10 generations old), which melanized to a greater extent than younger C. neoformans cells $(0-2$ generations old). Helal et al., which includes one of us, presented the first description that pan-antigens displayed on the cell

\section{REFERENCES}

Gow, N., Latge, J., and Munro, C. (2017). "The fungal cell wall: structure, biosynthesis, and function," in The Fungal Kingdom, eds J. Heitman, B. Howlett, P. Crous, E. Stukenbrock, T. James, and N. Gow (Washington, DC: ASM Press), 267-292. doi: 10.1128/microbiolspec.FUNK0035-2016

Conflict of Interest: The authors declare that the research was conducted in the absence of any commercial or financial relationships that could be construed as a potential conflict of interest. surface of pathogenic fungi can effectively be targeted with radioimmunotherapy. The authors described the ability of a radiolabeled anti-(1-3)- $\beta$-D-glucan antibody to specifically target Blastomyces dermatitidis in vitro and in vivo. Furthermore, this specific radioimmunotherapy selectively killed $B$. dermatitidis under both in vitro and in vivo conditions.

In conclusion, this themed collection enhances our knowledge of the diverse functions of the fungal cell wall in host-pathogen interactions, and the papers particularly highlighted potential targets and methods for antifungal development, reinforcing the relevance of studies focused on elucidating the biology of the fungal cell wall.

\section{AUTHOR CONTRIBUTIONS}

FA drafted the Editorial while JN and GN-V contributed to editing. All authors conceived and designed the work and provided final approval of the version to be published.

The handling editor declared a shared affiliation with one of the authors, FA, at time of review.

Copyright (C) 2020 Almeida, Nosanchuk and Niño-Vega. This is an open-access article distributed under the terms of the Creative Commons Attribution License (CC BY). The use, distribution or reproduction in other forums is permitted, provided the original author(s) and the copyright owner(s) are credited and that the original publication in this journal is cited, in accordance with accepted academic practice. No use, distribution or reproduction is permitted which does not comply with these terms. 